\title{
Expansion of tissue-resident CD8+ $T$ cells and CD4+ Th17 cells in the nasal mucosa following mRNA COVID-19 vaccination
}

Aloysious Ssemaganda ${ }^{1}$, Huong Mai Nguyen ${ }^{1}$, Faisal Nuhu ${ }^{1}$, Naima Jahan ${ }^{1}$, Catherine M. Card ${ }^{1,2}$, Sandra Kiazyk ${ }^{1,2}$, Giulia Severini ${ }^{1}$, Yoav Keynan ${ }^{1}$, Ruey-Chyi Su ${ }^{1,2}$, Hezhao Ji ${ }^{1,2}$, Bernard Abrenica ${ }^{2}$, Paul J. McLaren ${ }^{1,2}$, T. Blake Ball ${ }^{1,2}$, Jared Bullard ${ }^{1,3,4}$, Paul Van Caeseele ${ }^{1,3}$, Derek Stein ${ }^{1,3}$, Lyle R. McKinnon ${ }^{1,2,5}$

${ }^{1}$ Department of Medical Microbiology and Infectious Diseases, University of Manitoba, Winnipeg, MB, Canada.

2 JC Wilt Infectious Diseases Research Centre, National Microbiology Laboratory, Public Health Agency of Canada, Winnipeg, MB, Canada.

${ }^{3}$ Cadham Provincial Laboratory, Winnipeg, MB, Canada.

${ }^{4}$ Department of Pediatrics \& Child Health, University of Manitoba, Winnipeg, MB, Canada.

${ }^{5}$ Centre for the AIDS Programme of Research in South Africa (CAPRISA), Durban, South Africa.

Corresponding Author: Dr. Lyle McKinnon, Department of Medical Microbiology and Infectious Diseases, 504-745 Bannatyne Ave, University of Manitoba, Winnipeg, MB, Canada. R3E 0J9. Phone: (204) 975-7708. Email: lyle.mckinnon@umanitoba.ca

Word count, abstract: 150 .

Word count, main text: 1,503 . 


\begin{abstract}
Vaccines against SARS-CoV-2 have shown high efficacy in clinical trials, yet a full immunologic characterization of these vaccines, particularly within the upper respiratory tract, remains lacking. We enumerated and phenotyped $\mathrm{T}$ cells in nasal mucosa and blood before and after vaccination with the Pfizer-BioNTech COVID-19 vaccine $(\mathrm{n}=21)$. Tissue-resident memory $($ Trm) CD8+ T cells expressing CD69+CD103+ expanded $\sim 12$ days following the first and second doses, by 0.31 and $0.43 \log _{10}$ cells per swab respectively ( $\mathrm{p}=0.058$ and $\mathrm{p}=0.009$ in adjusted linear mixed models). CD69+CD103+CD8+ T cells in the blood decreased post-vaccination. Similar increases in nasal CD8+CD69+CD103- T cells were observed, particularly following the second dose. CD4+ Th17 cells were also increased in abundance following both doses. Following stimulation with SARS-CoV-2 spike peptides, CD8+ T cells increased expression of CD107a and CD154. These data suggest that nasal T cells may be induced and contribute to the protective immunity afforded by this vaccine.
\end{abstract}




\section{Main text}

The COVID-19 pandemic has led to substantial mortality and caused major global disruption. At least six vaccines have demonstrated efficacy in preventing severe disease associated with SARS-CoV-2 infection ${ }^{1}$. While this is an impressive achievement guided by early immunogenicity data, a full understanding of how these novel vaccines interact with the host immune system is lacking. This is particularly the case in tissues such as the upper respiratory tract (URT), which are often more challenging to sample than peripheral blood ${ }^{2}$. Here we focus on characterizing nasal mucosal $\mathrm{T}$ cells in healthy individuals pre- and post-vaccination with the BNT162b2 mRNA-nanoparticle vaccine developed by Pfizer-BioNTech ${ }^{3}$.

Natural history studies of SARS-CoV-2 infection demonstrate significant cell-mediated and humoral immune responses ${ }^{4-7}$, which in combination lead to a substantial decrease in re-infection risk $^{8}$. These responses can be readily detected for $>8$ months post-infection ${ }^{9}$, and appear to have a long halflife. In non-human primates, CD8+ T cell depletion led to increased susceptibility to re-infection ${ }^{10}$. Studies that assess the quality and quantity of immune responses induced by vaccination in humans are beginning to emerge ${ }^{11-16}$, adding depth to what was generated in early phase clinical trials. While these studies suggest a high magnitude of humoral and cell-mediated immunity to SARS-CoV-2 ${ }^{17,18}$, less is known regarding tissue and mucosal immunity induced by these vaccines, particularly in the URT, the primary site of viral entry.

We developed an ex vivo flow cytometry-based assay to enumerate and profile immune cells isolated from nasopharyngeal (NP) swabs used for SARS-CoV-2 diagnostic testing amongst healthy volunteers. We pilot-tested several types of NP swabs to gauge optimal immune cell recovery. Strikingly, CD45+ immune cells were recovered from a certain type of swab (Flexible minitip flocked swab from BD), while CD326+ epithelial cells were the main cell type recovered from two other swabs that we tested, including the Copan FLOQ swab (Figure 1A \&B). In a pilot study (n=8), a median of 3,082 (IQR: 2,351-6,168) CD45+ cells were recovered per BD swab, 80\% of which were CD3+ T cells. Using CD69 and CD103 as markers of tissue-resident memory (Trm) T cells ${ }^{19,20}$, virtually all CD8+ (>90\%) and variable proportions of CD4+ T cells were Trm (10-80\%; Figure 1C).

We enrolled healthy volunteers scheduled to receive the BNT162b2 mRNA vaccine ( $\mathrm{n}=21$, Supplemental Table 1), and collected baseline, pre-vaccine samples followed by peak response samples $\sim 12$ days after the first (visit 2) and second (visit 3) doses. Participants were a median age of 40 (IQR: 31-50) and predominantly female (14/21). Most participants (19/21) received influenza vaccination in the previous few months, and two participants had prior COVID-19 infection. The median body mass index (BMI) was 24 (IQR: 22-29). Reporting of underlying medical conditions 
included allergies and/or asthma $(n=6)$, type II diabetes $(n=2)$, hypertension $(n=2)$, and autoimmunity $(\mathrm{n}=1)$.

We analyzed both frequency of parent populations and total cell abundance, the latter by maximizing acquisition of cells from each swab; measuring abundance is critical as cell abundance can vary within mucosa across orders of magnitude ${ }^{21,22}$. We observed significant increases in nasal CD8+ Trm at both peak vaccine response time points (Figure 2A). In a linear mixed model adjusted for age, sex, BMI, prior COVID-19, influenza vaccination, and self-reported vaccine side effects, the number of CD8+ Trm increased by 0.31 and $0.44 \log _{10}$ cells per nasal swab following the first and second vaccine dose, respectively ( $\mathrm{p}=0.058$ and $\mathrm{p}=0.009)$. No increases in CD4+ Trm defined by CD103 and/or CD69 expression were observed (Figure 2B). The absolute number of nasal CD8+CD69+CD103- T cells also increased at visit 2 and particularly at visit 3, by 0.06 and $0.48 \log _{10}$ cells/swab, respectively (adjusted $\mathrm{p}=0.7$ and $\mathrm{p}=0.004$ for visits 2 and 3). The proportion of nasal CD8 $+\mathrm{T}$ cells that were Trm also increased, although not significantly ( $\mathrm{p}=0.09$ from visit 2 versus baseline, Supplemental Figure 1AB). Interestingly, the low proportion of CD69+CD103+ T cells in the blood significantly declined at the peak vaccine response time points, suggesting that these cells may be migrating out of the blood into tissues (Figure 2C-D). We also observed that CD4+ T cells co-expressing the Th17 markers CD161 and CCR6 increased by 0.40 and $0.45 \log _{10}$ cells/nasal swab at the peak vaccine response time points compared to baseline (adjusted $\mathrm{p}=0.017$ and $\mathrm{p}=0.008$, respectively) (Figure 2E). No increases in nasal Tfh cells were observed following either vaccine dose (Figure 2F). Similar increases were observed in analyses stratified by age and sex (Supplemental Table 2), and excluding participants with prior COVID-19 infection did not meaningfully change any outcomes.

Studies of other vaccines suggest that peak vaccine-elicited $T$ cells can be captured by activation markers such as Ki67, HLA-DR, and CD38, which peak at 10-14 days following yellow fever and smallpox vaccination ${ }^{23}$. In our study, we did not observe any changes in the proportion of CD4+ or CD8+ T cells co-expressing HLA-DR and CD38, or any upregulation of Ki-67, in either nasal or blood samples (Supplemental Figure 2).

We further analysed our flow data using the t-distributed stochastic neighbour embedding (tSNE), a dimensionality reduction method. Several substantial shifts in CD45+ nasal immune cell clusters were evident from baseline to visit 2, and from visit 2 to 3 (Supplemental Figure 3A). These included a step-wise shift in CD8+ T cell populations across the three time points, but also shifts in CD3- and CD3+CD4-CD8- cells. In support of this, the abundance of CD45+, CD3+, and CD8+ T cells all increased at visit 3, compared to baseline (Supplementary Figure 3B-G). While it was difficult to distinguish which phenotypic markers flow panel explained the observed divergent 
clustering patterns, these data suggest that multiple subsets of nasal immune cells may increase in number following COVID-19 vaccination.

To further assess the antigen-specificity of nasal T cells, we enrolled vaccinated individuals at 2 months following their second Pfizer-BNT dose, and either stimulated the NP swab cells with overlapping SARS-CoV-2 spike protein peptide pools $(n=15)$, or left cells in media unstimulated $(n=7)$. Stimulated samples had increased expression of the cytotoxic marker, CD107a $(p=0.016)$ and the activation marker CD154 ( $\mathrm{p}=0.069$, Figure 3). Similar trends were observed for TNF- $\alpha$ and perforin, though these were not statistically significant.

To understand the relationship between antibody titres and nasal $\mathrm{T}$ cells, we quantified levels of SARS-CoV-2 spike-specific IgG antibodies in plasma. Antibody titres increased at visit 2 (median titre 163, IQR: 67-376), and more dramatically at visit 3 (median 2,185, IQR: 826-3,652; Figure 4A). Only one of two participants with prior COVID-19 was positive for SARS-CoV-2 nucleocapsid IgG (Figure 4B). We next correlated antibody titres to the number of nasal CD8+ Trm and CD4+ Th17 at visit 3. Nasal CD8+ Trm and CD4+ Th17 increases were similar in participants with varying increases in spike titres, with no correlations observed (Figure 4C-D). These data suggest that tissue $\mathrm{T}$ cell responses to vaccination may be independent of the antibody response and may provide an additional layer of immunity against SARS-CoV-2 infection.

Trm are a vital form of surveillance in mucosal tissues, where they can mount rapid immune responses to pathogens upon re-exposure. In animal models, T cells at peak activation up-regulate homing markers that distribute these cells to relevant tissues ${ }^{24}$, where a high proportion is retained for extended periods as a form of sentinel surveillance against re-infection ${ }^{25}$. Trm have been more difficult to study in humans, given challenges in sampling the hard-to-reach tissues and in proving residency ${ }^{26,27}$. Our data suggest that certain types of NP swabs commonly used for COVID-19 diagnostics are effective in recovering immune cells from nasal tissue. Multiple populations of nasal CD4+ and CD8+ T cells increased in frequency by $\sim 0.5$ orders of magnitude following vaccination with the BNT162b2 mRNA vaccine. This is a critical observation, as most human mucosal COVID-19 studies have focused either on antibodies ${ }^{28}$ or lung $\mathrm{T}$ cells ${ }^{29,30}$; while a tissue population, the latter may not represent the front-line defense against infection. It has been hypothesized that Trm may be the most effective form of $\mathrm{T}$ cell response against SARS-CoV-2 infection ${ }^{31}$, capable of preventing viral dissemination beyond the URT, where more virus-induced damage can occur ${ }^{32}$. Data from mice have suggested that parenteral vaccination induces nasal Th17 Trm that protect against bacterial colonization $^{33,34}$. However, our study is the first, to our knowledge, to suggest nasal $\mathrm{T}$ cells may be induced by parenteral vaccination against COVID-19. 
Our study has some limitations. We enrolled a relatively small sample size, restricting our ability to extensively stratify our data. The age range does not include the elderly, a group most in need of protection by COVID-19 vaccination ${ }^{35}$. The immune cell recovery from NP swabs, while sufficient for phenotyping and enumeration, is challenging for extensive functional characterization, in particular in assays that required splitting samples for paired analyses.

In summary, nasal T cell studies are feasible in humans, but this is highly dependent on the type of NP swab that is used. A high proportion of nasal T cells express markers of tissue residency, and these cells increase significantly in number and function following SARS-CoV-2 mRNA immunization. Further work should be performed to understand the role that local $\mathrm{T}$ cells may play in the protection against SARS-CoV-2 infection. 


\section{Acknowledgements}

This work was funded by the Bill and Melinda Gates Foundation and the Canadian Institutes of Health

Research. Special thanks to the study volunteers whose time and willingness to provide repeated specimens made this project possible.

\section{Author contributions}

AS, DS, LRM: Conceptualized the study, designed the experiments and analysed data.

LRM, TBB, JB, PVC: Overall study supervision and coordination.

CMC, SK, YK, RS, HJ, PJM, TBB, JB, PVC, DS: Provided intellectual input into study design.

NJ, GS, BA: Enrolled study participants, carried out sampling and/or collected and entered data.

AS, HMN, FN, DS: Performed experiments.

AS, LRM: Wrote the initial draft manuscript.

All authors reviewed and approved the submitted manuscript. 


\section{References}

1. Carvalho, T., Krammer, F. \& Iwasaki, A. The first 12 months of COVID-19: a timeline of immunological insights. Nat. Rev. Immunol. 1-12 (2021). doi:10.1038/s41577-021-00522-1

2. Sette, A. \& Crotty, S. Adaptive immunity to SARS-CoV-2 and COVID-19. Cell 183, 1354 (2021).

3. Polack, F. P. et al. Safety and Efficacy of the BNT162b2 mRNA Covid-19 Vaccine. N. Engl. J. Med. 383, 2603-2615 (2020).

4. Braun, J. et al. SARS-CoV-2-reactive T cells in healthy donors and patients with COVID-19. Nature 587, 270-274 (2020).

5. Grifoni, A. et al. Targets of T Cell Responses to SARS-CoV-2 Coronavirus in Humans with COVID-19 Disease and Unexposed Individuals. Cell 181, 1489-1501.e15 (2020).

6. Mateus, J. et al. Selective and cross-reactive SARS-CoV-2 T cell epitopes in unexposed humans. Science 370, 89-94 (2020).

7. Le Bert, N. et al. SARS-CoV-2-specific T cell immunity in cases of COVID-19 and SARS, and uninfected controls. Nature 584, 457-462 (2020).

8. D, W. et al. SARS-CoV-2 responsive T cell numbers are associated with protection from COVID-19: A prospective cohort study in keyworkers. medRxiv 2020.11.02.20222778 (2020). doi:10.1101/2020.11.02.20222778

9. Dan, J. M. et al. Immunological memory to SARS-CoV-2 assessed for up to 8 months after infection. Science eabf4063 (2021). doi:10.1126/science.abf4063

10. McMahan, K. et al. Correlates of protection against SARS-CoV-2 in rhesus macaques. Nature 590, 630-634 (2021).

11. Muik, A. et al. Neutralization of SARS-CoV-2 lineage B.1.1.7 pseudovirus by BNT162b2 vaccine-elicited human sera. Science 371, 1152-1153 (2021).

12. DiPiazza, A. T., Graham, B. S. \& Ruckwardt, T. J. T cell immunity to SARS-CoV-2 following natural infection and vaccination. Biochem Biophys Res Commun 538, 211-217 (2021).

13. Wang, Z. et al. mRNA vaccine-elicited antibodies to SARS-CoV-2 and circulating variants. Nature 1-7 (2021). doi:10.1038/s41586-021-03324-6

14. Zhou, D. et al. Evidence of escape of SARS-CoV-2 variant B.1.351 from natural and vaccineinduced sera. Cell (2021). doi:10.1016/j.cell.2021.02.037

15. Tarke, A. et al. Negligible impact of SARS-CoV-2 variants on CD4 + and CD8 + T cell reactivity in COVID-19 exposed donors and vaccinees. bioRxiv 2021.02.27.433180 (2021). doi:10.1101/2021.02.27.433180

16. Manisty, C. et al. Antibody response to first BNT162b2 dose in previously SARS-CoV-2infected individuals. Lancet 397, 1057-1058 (2021).

17. Shrotri, M. et al. T cell response to SARS-CoV-2 infection in humans: A systematic review. PLoS ONE 16, e0245532 (2021).

18. Carsetti, R. et al. Different Innate and Adaptive Immune Responses to SARS-CoV-2 Infection of Asymptomatic, Mild, and Severe Cases. Front Immunol 11, 610300 (2020).

19. Mackay, L. K. et al. The developmental pathway for CD103(+)CD8+ tissue-resident memory $\mathrm{T}$ cells of skin. Nat. Immunol. 14, 1294-1301 (2013).

20. Casey, K. A. et al. Antigen-independent differentiation and maintenance of effector-like resident memory T cells in tissues. J. Immunol. 188, 4866-4875 (2012).

21. McKinnon, L. R. et al. Characterization of a human cervical CD4+ T cell subset coexpressing multiple markers of HIV susceptibility. J. Immunol. 187, 6032-6042 (2011).

22. Arnold, K. B. et al. Increased levels of inflammatory cytokines in the female reproductive tract are associated with altered expression of proteases, mucosal barrier proteins, and an influx of HIV-susceptible target cells. Mucosal Immunol 9, 194-205 (2016). 
23. Miller, J. D. et al. Human effector and memory CD8+ T cell responses to smallpox and yellow fever vaccines. Immunity 28, 710-722 (2008).

24. Masopust, D. et al. Dynamic T cell migration program provides resident memory within intestinal epithelium. J. Exp. Med. 207, 553-564 (2010).

25. Masopust, D. \& Schenkel, J. M. The integration of T cell migration, differentiation and function. Nat. Rev. Immunol. 13, 309-320 (2013).

26. Buggert, M. et al. Identification and characterization of HIV-specific resident memory CD8+ T cells in human lymphoid tissue. Sci Immunol 3, eaar4526 (2018).

27. Kumar, B. V. et al. Human Tissue-Resident Memory T Cells Are Defined by Core Transcriptional and Functional Signatures in Lymphoid and Mucosal Sites. Cell Rep 20, 29212934 (2017).

28. Isho, B. et al. Persistence of serum and saliva antibody responses to SARS-CoV-2 spike antigens in COVID-19 patients. Sci Immunol 5, (2020).

29. Wauters, E. et al. Discriminating mild from critical COVID-19 by innate and adaptive immune single-cell profiling of bronchoalveolar lavages. Cell Res 31, 272-290 (2021).

30. Grant, R. A. et al. Circuits between infected macrophages and T cells in SARS-CoV-2 pneumonia. Nature 590, 635-641 (2021).

31. Jarjour, N. N., Masopust, D. \& Jameson, S. C. T Cell Memory: Understanding COVID-19. Immunity 54, 14-18 (2021).

32. Lipsitch, M., Grad, Y. H., Sette, A. \& Crotty, S. Cross-reactive memory T cells and herd immunity to SARS-CoV-2. Nat. Rev. Immunol. 20, 709-713 (2020).

33. O'Hara, J. M. et al. Generation of protective pneumococcal-specific nasal resident memory CD4+ T cells via parenteral immunization. Mucosal Immunol 13, 172-182 (2020).

34. Allen, A. C. et al. Sustained protective immunity against Bordetella pertussis nasal colonization by intranasal immunization with a vaccine-adjuvant combination that induces IL-17-secreting TRM cells. Mucosal Immunol 11, 1763-1776 (2018).

35. Koff, W. C. et al. Development and deployment of COVID-19 vaccines for those most vulnerable. Sci Transl Med 13, (2021). 
(A)

BD Mini flex-tip

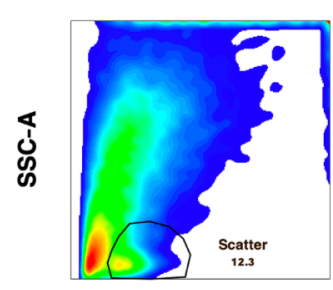

Copan FLOQ

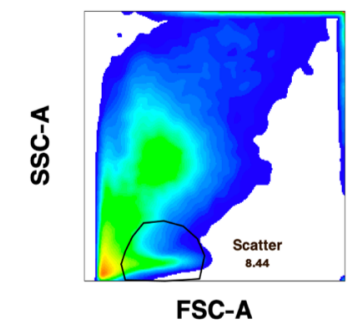

FSC-A
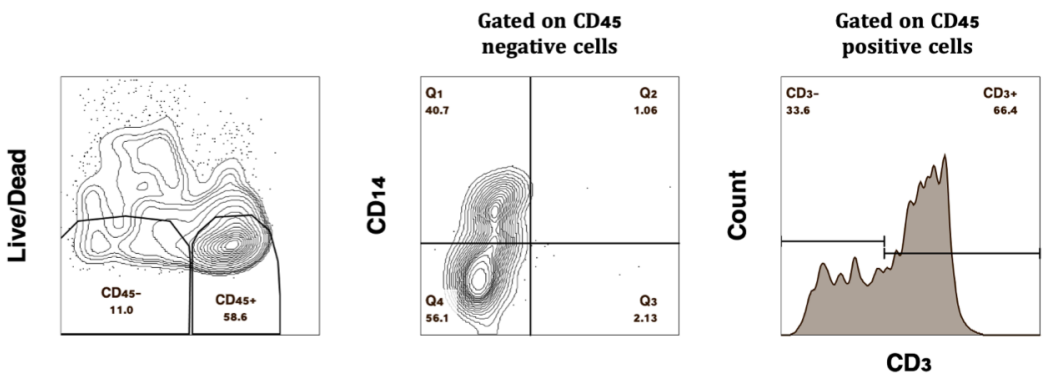

(B)
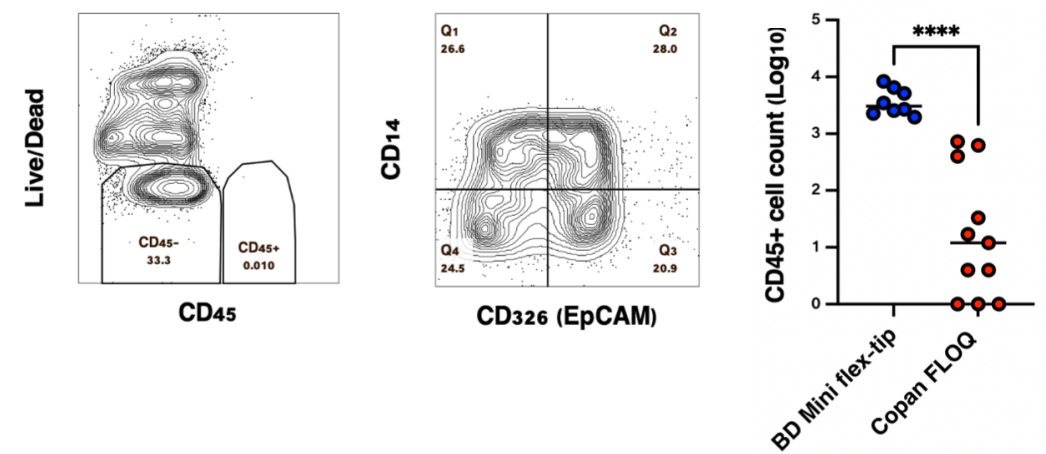

Th17
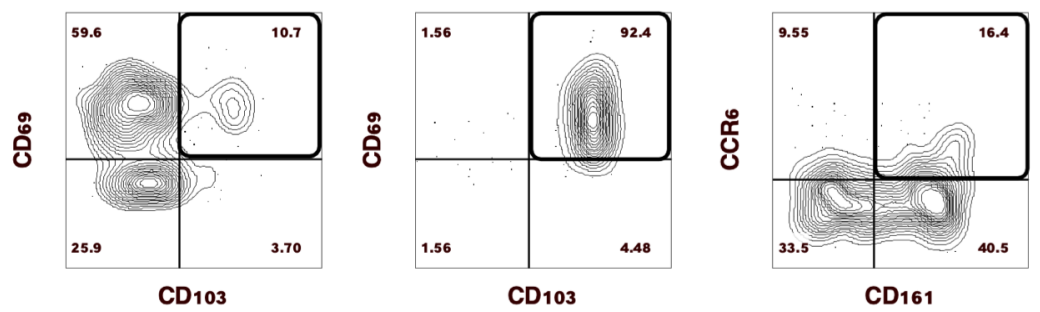

$\mathrm{CD} 4$
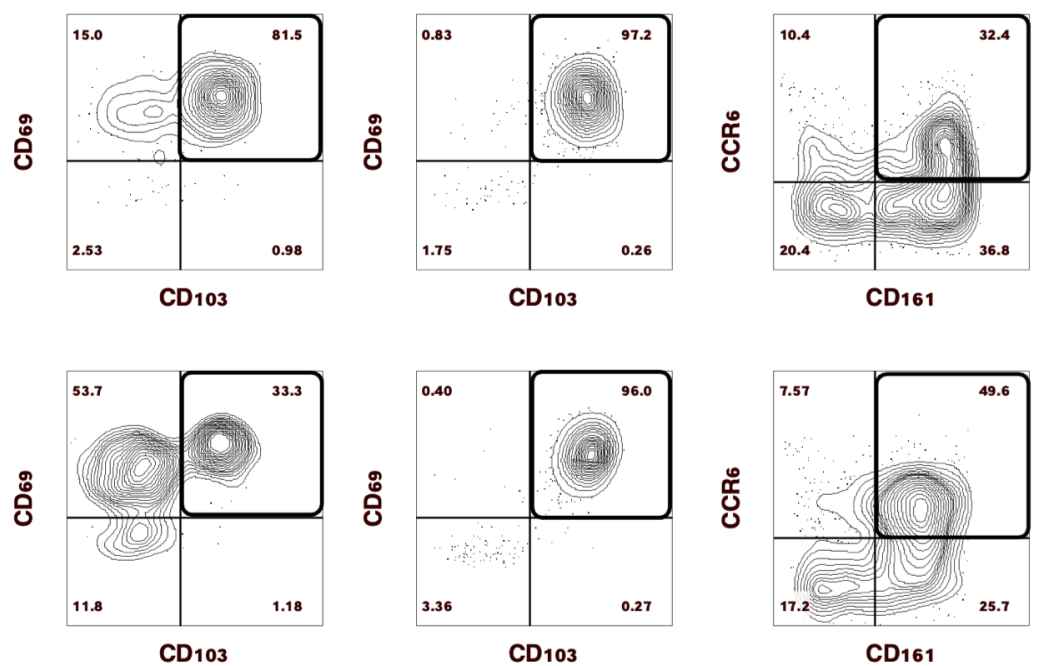

CD161

Figure 1: Comparison of SARS-CoV-2 diagnostic testing nasopharyngeal (NP) swabs for immune cell recovery. Representative plots of CD45+ nasal immune cells isolated from (A) BD Mini Flex-tip swabs, majority of which ( 40-90\%) were CD3+ and Copan FLOQ swabs from which epithelial (CD326 $($ EpCAM+) cells were the main cell type recovered. (B) Swab brand comparison of CD45+ immune cell recovery. (C) Representative plots of CD3+ T cell subsets isolated from BD Mini-tip flexible swabs. Majority of CD4+ and CD8+ T cells were Trm based on the expression of CD69 and CD103, while 16-50\% of CD4 T cells were Th17 based on the expression of CD161 and CCR6. (****P $>0.0001)$ 


\section{Nasal}

A

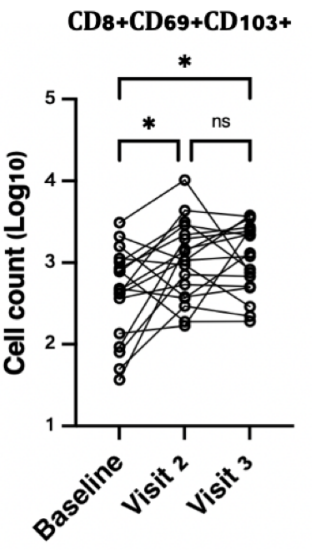

C

CD4+CD69+CD103+

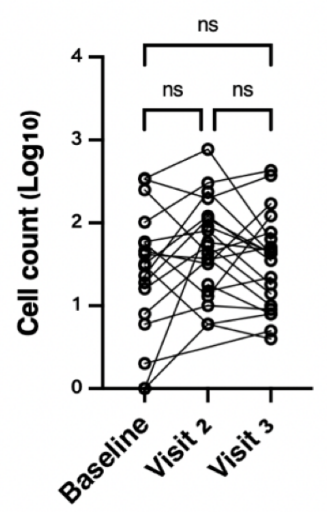

CD8+CD69+CD103-

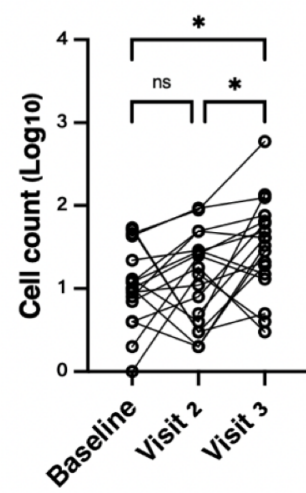

CD4+CD69+CD103-

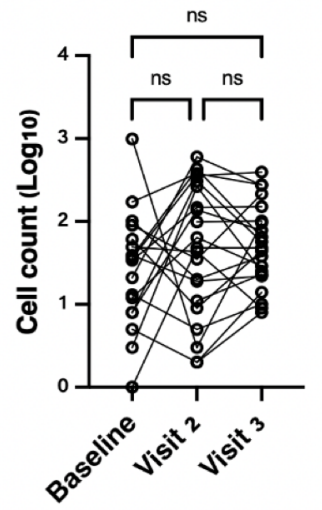

B

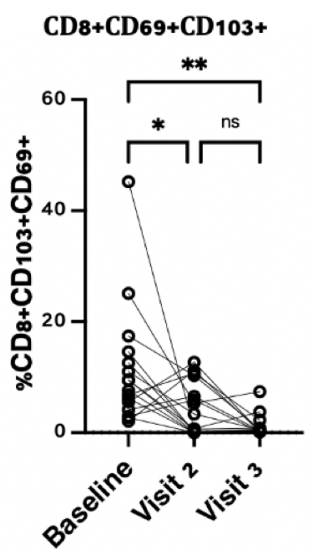

D

CD4+CD69+CD103+

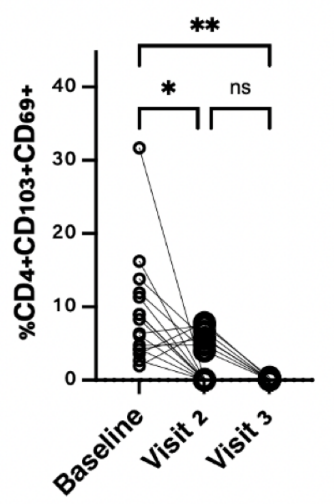

\section{Blood}

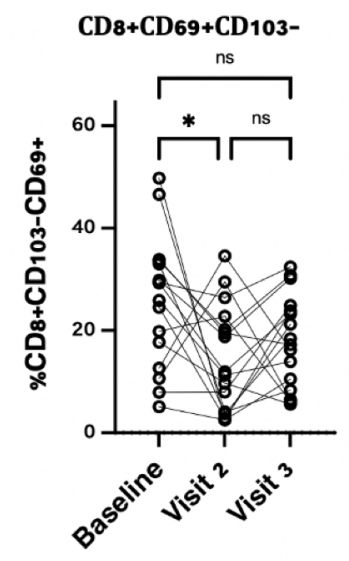

CD4+CD69+CD103-

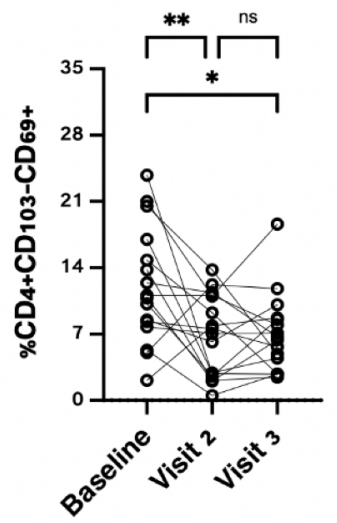

Nasal

E

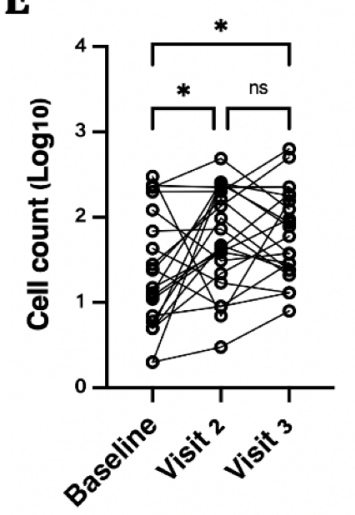

F

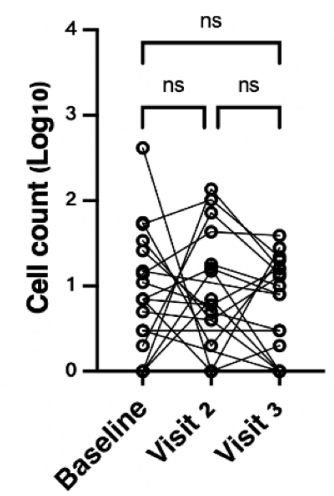

Figure 2: Nasal cell counts per NP swab and peripheral blood frequencies of $T$ cell subsets post SARS-CoV-2 vaccination. (A) Nasal CD8+CD69+CD103+ Trm significantly increased following the first and second vaccine dose while CD8+CD69+CD103- T cells increased significantly following the second vaccine dose. (B) No changes in nasal CD4 +CD69+CD103+ Trm (C) In blood, CD8+CD69+CD103+ Trm and (D) CD4+CD69+CD103+ decreased significantly at visit 2 and 3 compared to baseline. (E) Significant increases in Th17 $(\mathrm{CD} 4+\mathrm{CD} 161+\mathrm{CCR} 6+) \mathrm{T}$ cells at peak vaccine response time points while $(\mathrm{E})$ no changes were observed in Tfh $(\mathrm{CD} 4+\mathrm{CXCR} 5+\mathrm{PD} 1+)$ T cells. $\left({ }^{*} \mathrm{P}>0.01, * * \mathrm{P}>0.001\right.$, ns: non-significant). 
(A)

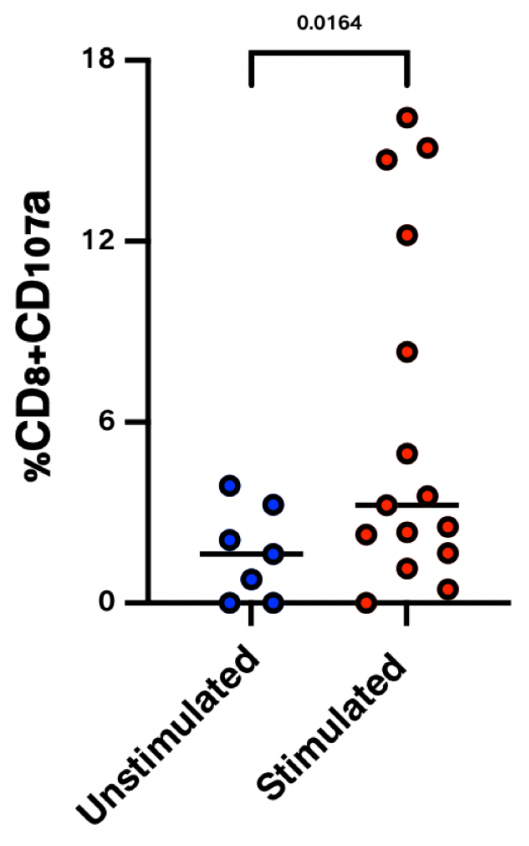

(D)

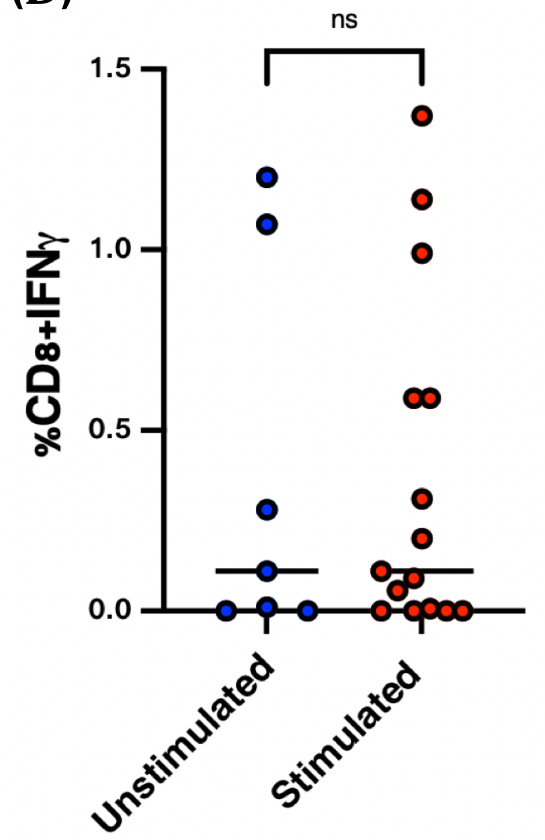

(B)

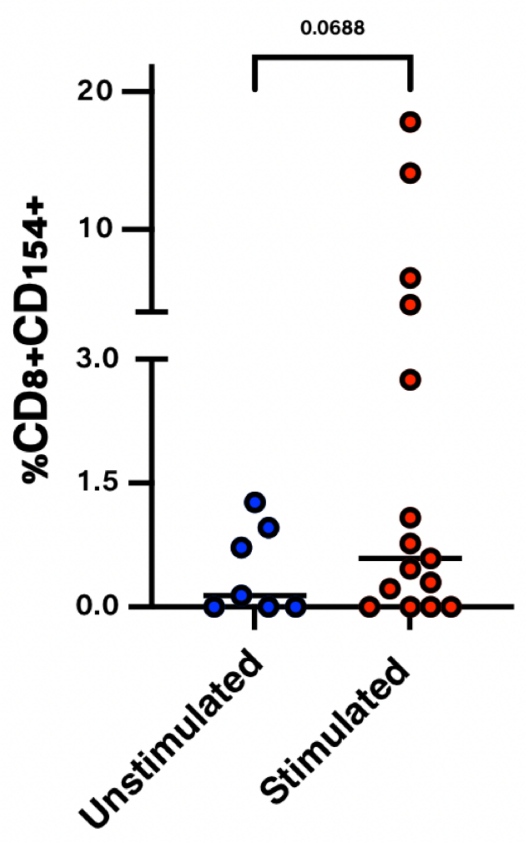

(C)

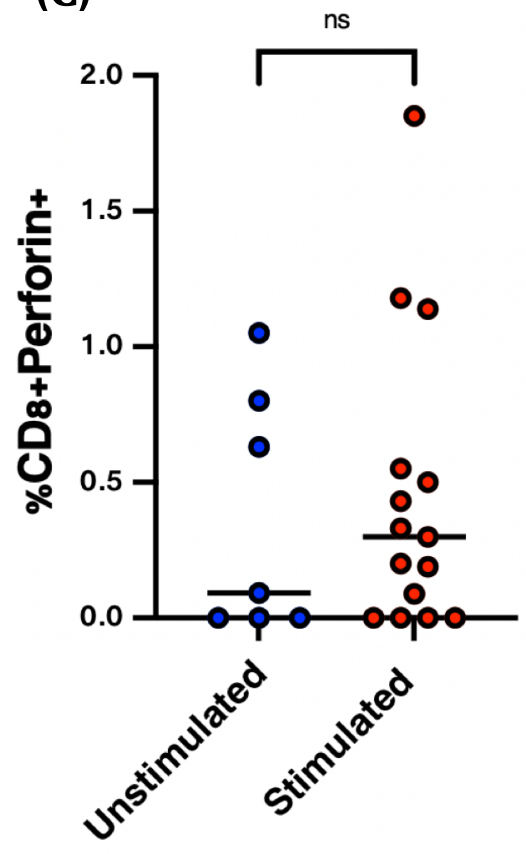

(E)

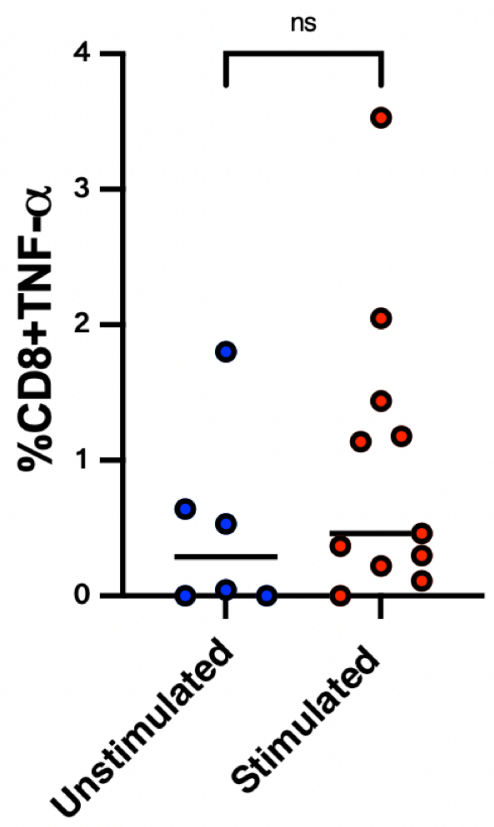

Figure 3: SARS-CoV-2 spike-specific CD8+ $T$ cell responses $\sim 2$ months post-vaccination. Nasal immune cells isolated from individuals, approximately 2 months post second vaccine dose, were stimulated ( $\mathrm{n}=15)$ with overlapping spike peptides (Red dots) or left unstimulated ( $\mathrm{n}=7)$ (Blue dots). Frequencies of (A) CD107a, (B) CD154, (C) Perforin, (D) TNF- $\alpha$ and (E) IFN- $\gamma$ antigen-specific CD8+ T cell responses. (ns: non-significant). 
A

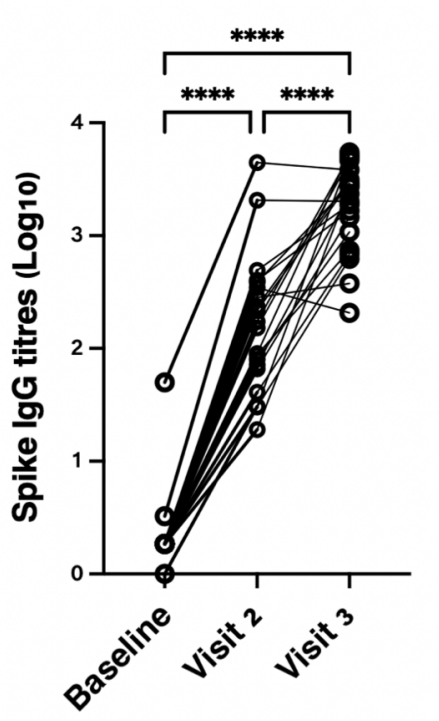

C

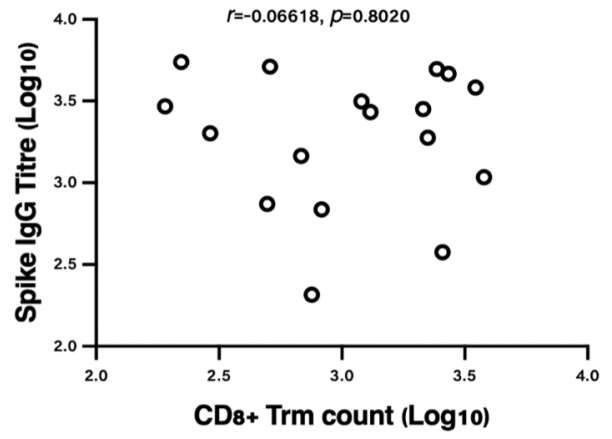

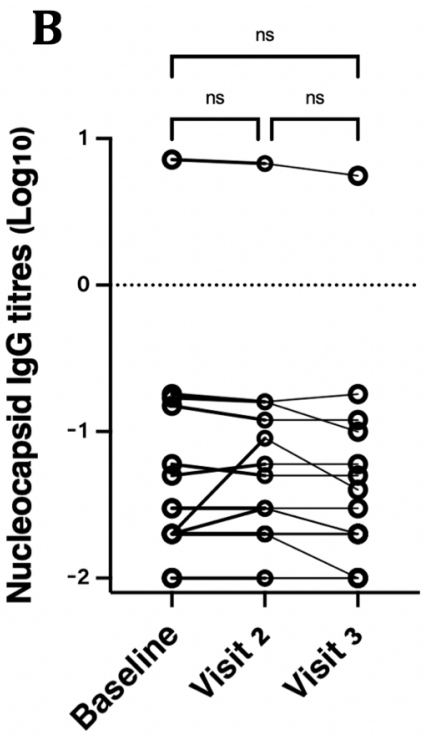

D

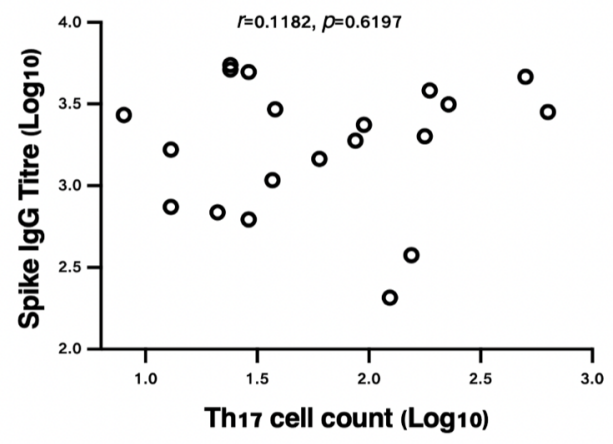

Figure 4: Plasma IgG antibody titres following SARS-COV-2 vaccination (A) spike-specific IgG titres and (B) nucleocapsid specific IgG titres. (C) Correlation of nasal CD8+ Trm and CD4+ Th17 T cell counts with spike antibody titres at visit 3, 12 days following the second SARS-COV-2 vaccine dose $(* * * * \mathrm{p}<0.0001$, ns: non-significant). 
Supplemental Table 1. Participant demographics and health status.

\begin{tabular}{|ll|}
\hline Variable & Study participants (n= 21) \\
\hline Age (median, IQR) & $40(31-50)$ \\
\hline Sex, female & $67 \%(14 / 21)$ \\
\hline Body mass index (BMI, median, IQR) & $24.4(21.5-28.9)$ \\
\hline Received 2020 fall flu vaccine & $90 \%(19 / 21)$ \\
\hline Diagnosed with COVID-19 in 2020 & $10 \%(2 / 21)$ \\
\hline Self-reported underlying medical conditions & Asthma/allergy $(6 / 21)$ \\
& Type 2 diabetes $(2 / 21)$ \\
& Hypertension $(2 / 21)$ \\
& Autoimmunity $(1 / 21)$ \\
\hline Dose 2 side effects & None $(5 / 21)$ \\
& Sore arm $(3 / 21)$ \\
& Moderate effects including nausea, fever, influenza-like \\
& symptoms (13/21) \\
\hline
\end{tabular}

Supplemental Table 2. Age and Sex-stratified analysis of CD8+ Trm and Th17 cells following SARS-CoV-2 vaccination

\begin{tabular}{|c|c|c|c|c|}
\hline \multicolumn{2}{|c|}{ Strata } & \multicolumn{3}{|c|}{ Outcome* (V3 vs baseline; beta \& 95\% CI) } \\
\hline & & $\begin{array}{c}\mathrm{CD} 8+ \\
\text { CD69+CD103+ }\end{array}$ & $\begin{array}{c}\text { CD8+ } \\
\text { CD69+CD103- }\end{array}$ & $\begin{array}{c}\text { CD4+ } \\
\text { CCR6+CD161+ }\end{array}$ \\
\hline \multicolumn{2}{|c|}{ Entire cohort } & $0.43(0.11-0.64)$ & $0.48(0.16-0.81)$ & $0.45(0.12-0.78)$ \\
\hline \multirow[t]{2}{*}{ Sex } & Males $(n=7)$ & $0.57(0.08-1.07)$ & $0.39(-0.11-0.90)$ & $0.38(-0.07-0.83)$ \\
\hline & Females $(n=14)$ & $0.39(0.00-0.78)$ & $0.51(0.11-0.90)$ & $0.49(0.08-0.90)$ \\
\hline \multirow[t]{3}{*}{ Age } & $\begin{array}{c}\leq 35 \text { years of age } \\
(n=8)\end{array}$ & $0.21(-0.30-0.71)$ & $0.22(-0.18-0.62)$ & $0.42(-0.14-0.98)$ \\
\hline & $\begin{array}{c}>35 \text { to }<50 \text { years } \\
\text { of age }(n=6)\end{array}$ & $0.85(0.68-1.02)$ & $1.00(0.56-1.44)$ & $0.55(0.18-0.91)$ \\
\hline & $\begin{array}{l}\geq 50 \text { years of age } \\
\quad(n=7)\end{array}$ & $0.45(-0.06-0.95)$ & $0.42(-0.09-0.94)$ & $0.41(-0.13-0.95)$ \\
\hline
\end{tabular}


Supplementary Table 3. List of flow cytometry antibodies/reagents

\begin{tabular}{|c|c|c|}
\hline Antibody/reagents & Catalogue No. & Brand \\
\hline Anti- Human CD326 (EpCAM) Brilliant Violet 650 (Clone 9C4) & 324226 & Biolegend \\
\hline Anti-Human CD38 BB515 (Clone HIT2) & 564498 & BD Horizon \\
\hline Anti- Human Perforin PerCP/Cyanine5.5 (Clone B-D48) & 353314 & Biolegend \\
\hline Anti- Human CD134 (OX40) PE/Cyanine7 (Clone Ber-ACT35) & 350012 & Biolegend \\
\hline Anti-Human CD69 PE/Cyanine5 (Clone FN50) & 310908 & Biolegend \\
\hline Anti-Human CD161 PE (Clone HP-3G10) & $12-1619-42$ & eBioscience \\
\hline Anti-Human Bcl-2 PE (Clone100) & 658708 & Biolegend \\
\hline Anti-Human CD196 (CCR6) BV421(Clone 11A9) & 562515 & BD Horizon \\
\hline $\begin{array}{l}\text { Anti-Human CD103 (Integrin } \alpha \text { E) Brilliant Violet } 605 \text { (Clone Ber- } \\
\text { ACT8) }\end{array}$ & 350218 & Biolegend \\
\hline Anti-Human HLA-DR BV650 (Clone G46-6) & 564231 & BD Horizon \\
\hline Anti-Human CD154 Brilliant Violet 711(Clone 24-31) & 310838 & Biolegend \\
\hline Anti-Human CD45 Brilliant Violet 785(Clone HI30) & 304048 & Biolegend \\
\hline Anti-Human CD8a APC/Cyanine7 (Clone RPA-T8) & 301016 & Biolegend \\
\hline Anti-Human CD3 Alexa Fluor ${ }^{\circledR} 700$ (Clone UCHT1) & 300424 & Biolegend \\
\hline Anti-Human Ki-67 Alexa Fluor® 647 (Clone B56) & 561126 & BD Pharmingen \\
\hline Anti-Human CXCR5 (CD185) BUV395(Clone RF8B2) & 740266 & BD OptiBuild \\
\hline Anti-Human CD4 BUV496 (Clone SK3) & 612936 & BD Horizon \\
\hline Anti-Human CD279 (PD-1) BUV737 (Clone EH12.1) & 612791 & BD Horizon \\
\hline LIVE/DEAD ${ }^{\mathrm{TM}}$ Fixable Aqua Dead Cell Stain Kit & L34957 & Invitrogen \\
\hline \multicolumn{3}{|c|}{ Antigen-specific staining antibodies } \\
\hline Anti-Human TNF- $\alpha$ FITC (Clone (MAb11) & $11-7349-41$ & eBioscience \\
\hline Anti-Human Perforin PerCP/Cyanine5.5 (Clone B-D48) & 353314 & Biolegend \\
\hline Anti-Human IFN- $\gamma$ PE/Cyanine7(Clone B27) & 557643 & BD Pharmingen \\
\hline Anti-Human CD107a PE-CF594 (Clone H4A3) & 562628 & BD Horizon \\
\hline Anti-Human IL-22 PE (Clone 22URTI) & $12-7229-42$ & eBioscience \\
\hline Anti-Human IL-17A BV421(Clone ) & N49-653 & BD Pharmingen \\
\hline Anti-Human CD8 BV650 (Clone RPA-T8) & 564231 & BD Horizon \\
\hline Anti-Human CD154 (CD40L) Brilliant Violet 711(Clone 24-31) & 310838 & Biolegend \\
\hline Anti-Human CD45 Brilliant Violet 785(Clone HI30) & 304048 & Biolegend \\
\hline Anti-Human CD3 APC-H7 (Clone: SK7) & 560176 & BD Pharmingen \\
\hline Anti-Human IL-2 APC-R700 (Clone MQ1-17H12) & 565136 & BD Horizon \\
\hline Anti-Human Ki-67 Alexa Fluor ${ }^{\circledR} 647$ (Clone B56) & 561126 & BD Pharmingen \\
\hline Anti-Human CD45RO BUV395(Clone UCHL1) & 564291 & BD Horizon \\
\hline Anti-Human CD4 BUV496 (Clone SK3) & 612936 & BD Horizon \\
\hline
\end{tabular}


A

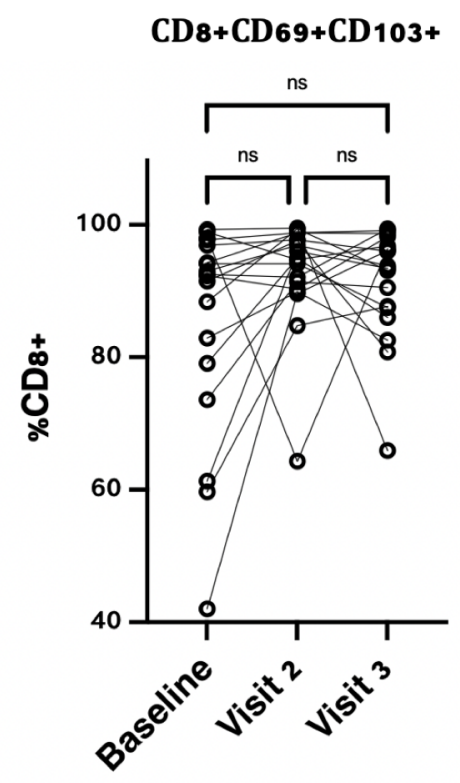

C

CD4+CD69+CD103+

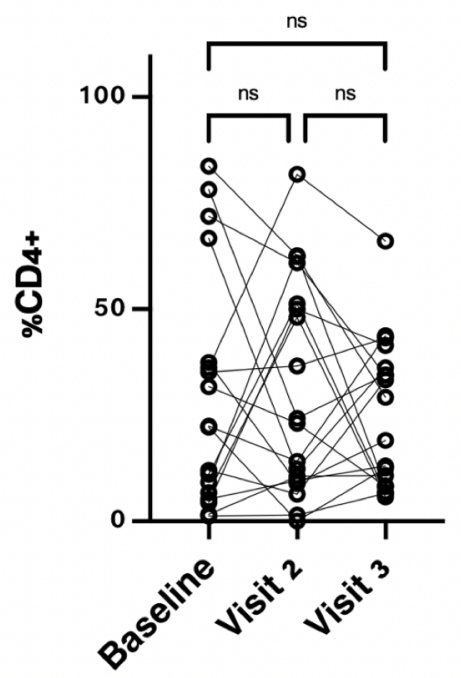

B

CD8+CD69+CD103-

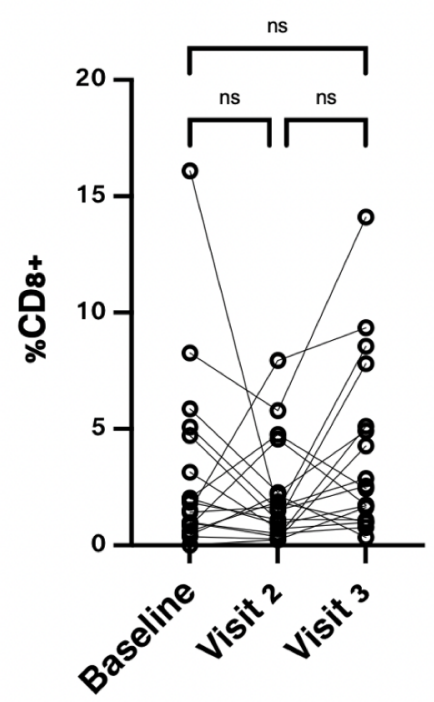

D

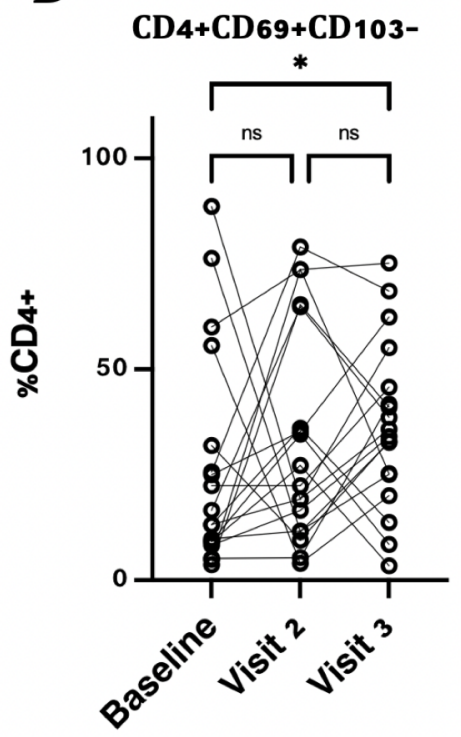

Supplementary Figure 1: Frequencies of nasal CD4 and CD8 Trm T cells post SARS-COV-2 vaccination. Proportions of (A) CD8+CD69+CD103+, (B) CD8+CD69+CD103-, (C) CD4+CD69+CD103+Trm and (D) CD4+CD69+CD103+ Trm $\mathrm{T}$ cells $(* \mathrm{P}>0.01$, ns: non-significant). 
(A)

\section{CD8+HLA-DR+CD38+}

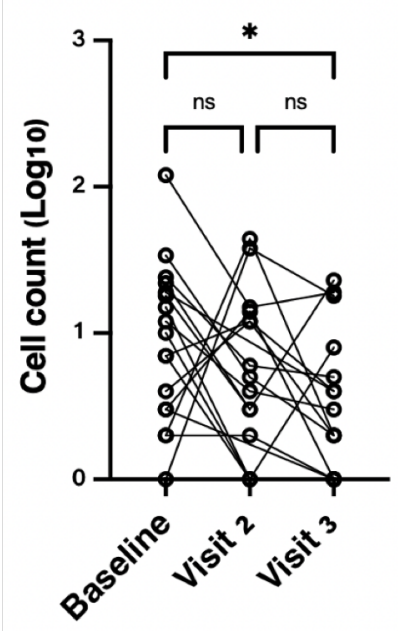

(B)

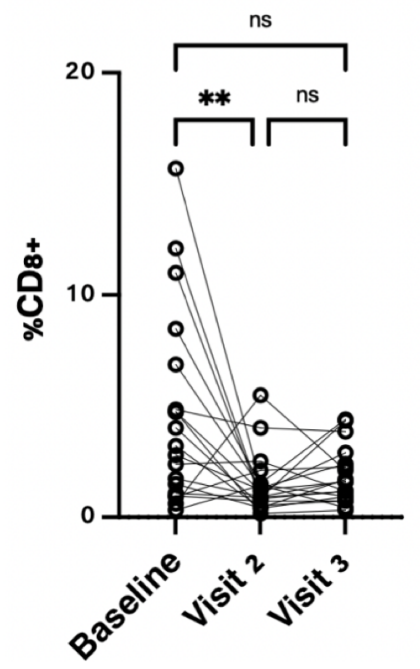

Nasal

CD8+Ki67+

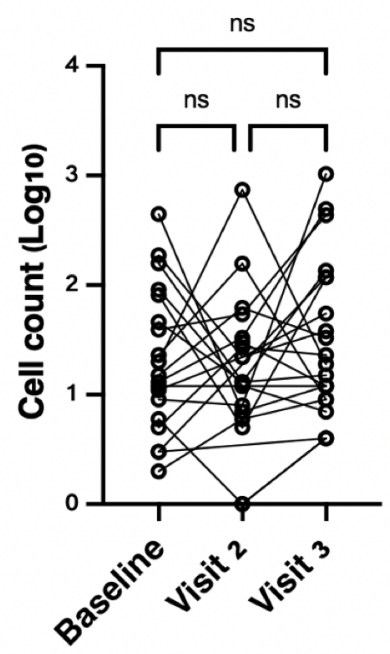

Blood

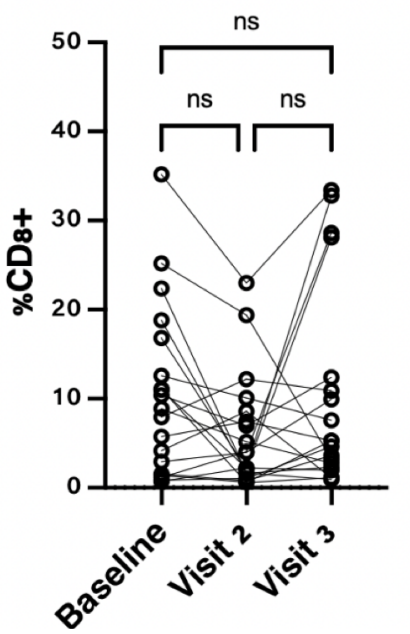

CD8+Perforin+
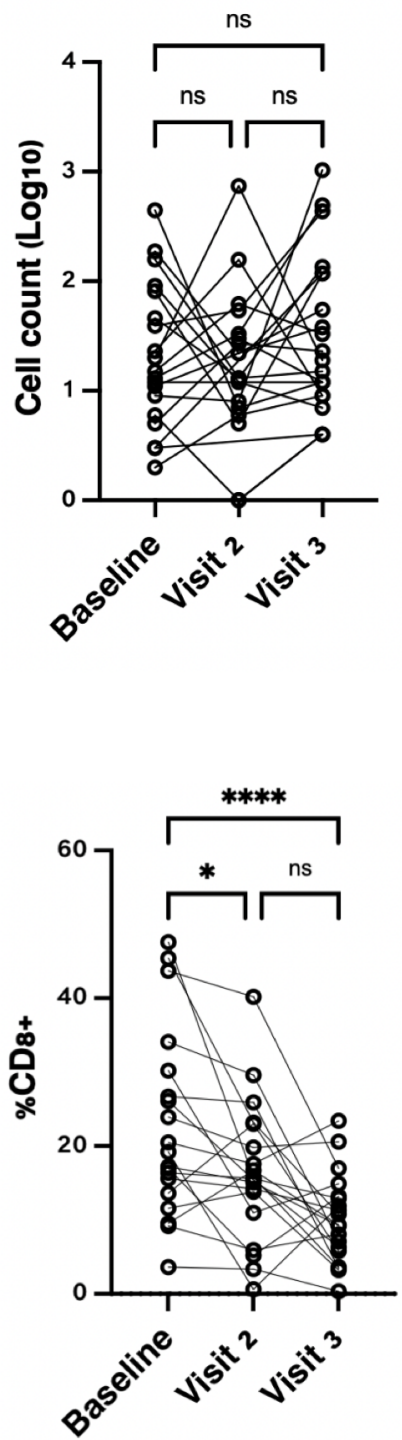

Supplementary Figure 2. CD8+ T cell activation defined by expression of HLA-DR and CD38, proliferation, based on the expression of Ki-67 and cytotoxicity, defined by the expression of perforin in cells isolated from (A) nasopharyngeal swabs and (B) peripheral blood amongst vaccinated individuals $(\mathrm{n}=21)$ following SARS-COV-2 vaccination $\left(* \mathrm{p}<0.01,{ }^{*} \mathrm{p}<0.001\right.$, $* * * * \mathrm{p}<0.0001$, ns: non-significant). 


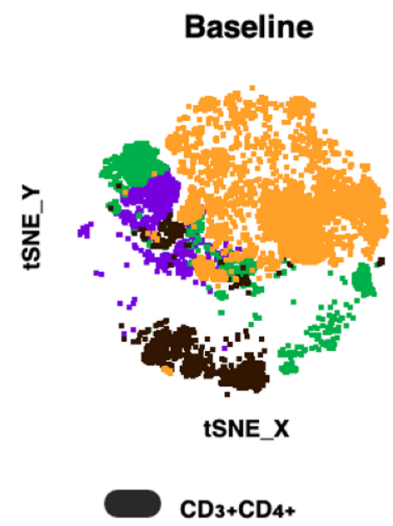

B

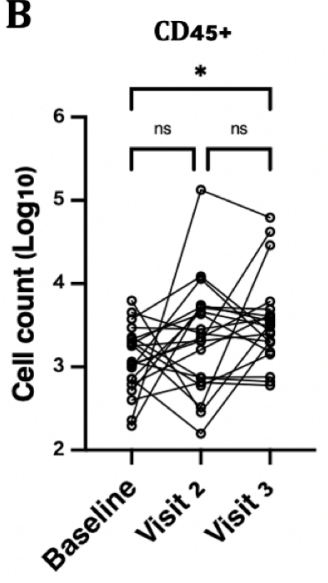

$\mathbf{E}$

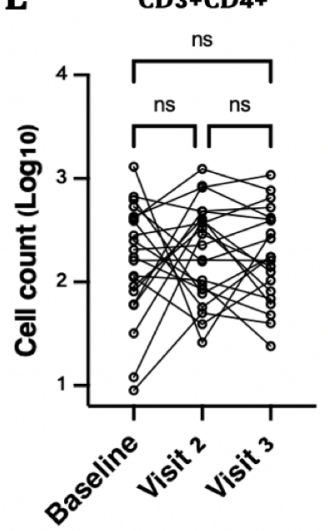

Visit 2

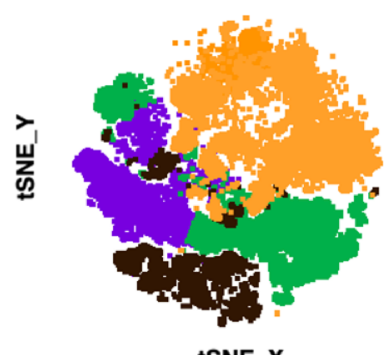

$\mathrm{CD}_{3}+\mathrm{CD}_{8}+$
Visit 3

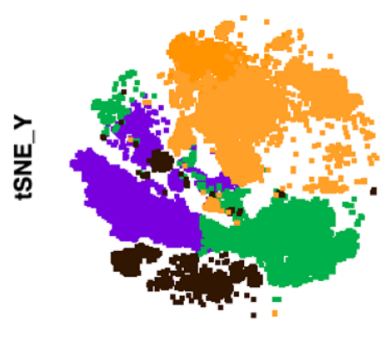

tSNE_X
C

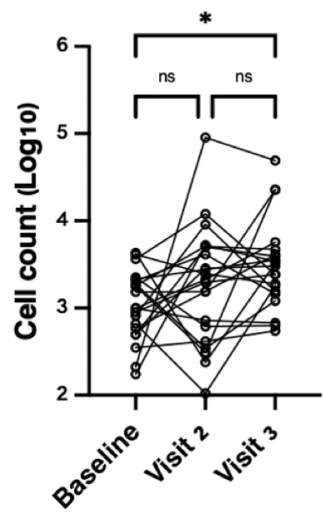

$\mathbf{F}$

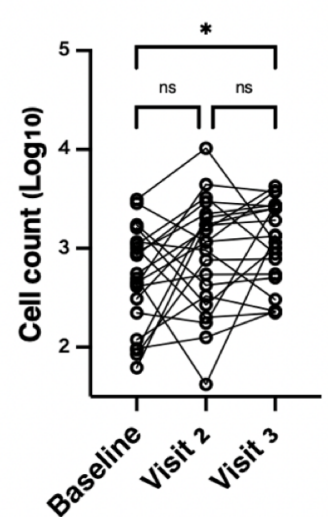

D $\mathrm{CD} 3+\mathrm{CD} 4-\mathrm{CD} 8-$

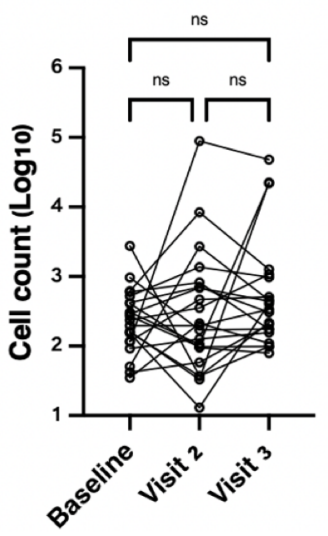

G CD3-CD4-CD8-

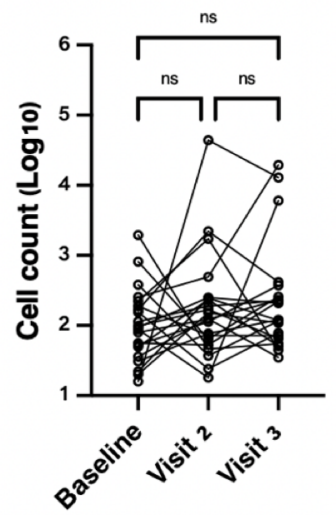

Supplementary Figure 3. Nasal immune cell clustering and cell counts per swab following SARS-COV-2 vaccination. (A) t-distributed stochastic neighbour embedding (tSNE) plots of integrated nasal CD45+ immune cells showing shifts in CD3+CD4+, CD3+CD8+, CD3-CD4CD8- and CD3+CD4-CD8- immune cell clusters from vaccinated individuals at baseline, visit 2 and visit 3. (B) $\mathrm{CD} 45+(\mathrm{C}) \mathrm{CD} 45+\mathrm{CD} 3+$ and $(\mathrm{F}) \mathrm{CD} 3+\mathrm{CD} 8+$ cells were significantly increased following the $2^{\text {nd }}$ vaccination dose while no changes were observed for (D) CD3+CD4-C8-, (E) CD3+CD4+ and (G) CD3-CD4-CD8- immune cell subsets (* $\mathrm{P}>0.01$, ns: non-significant). 
bioRxiv preprint doi:htths://fol.org/10.1101/2021.05.07.442971; this version posted May 9, 2021. The copyright holder for this preprint (which Supphefe available under aCC-BY-NC-ND 4.0 International license.

Study participants and ethics board approval. Study volunteers $(n=29)$ included staff enrolled at the Cadham Provincial Laboratory in Winnipeg, with ethics approval from the University of Manitoba Health Research Ethics Board (HREB) and the Public Health Agency of Canada (PHAC). Informed consent was obtained and a comprehensive socio-demographic and health assessment questionnaire administered to all study participants.

Sample collection and processing. In pilot work, we compared the BD Flex-mini and Copan FLOQ swabs. In the vaccine study participants, matched diagnostic pharyngeal (NP) swabs (BD Flex-mini) and peripheral blood samples (BD EDTA Vacutainer) were collected prior to vaccination (baseline), $\sim 12$ days after the first vaccine dose (visit 2), and $\sim 12$ days after the second dose (visit 3). Nasal cells were isolated from NP swabs which were inserted into the nose, rotated once $\left(360^{\circ}\right)$ and placed into a tube containing $3 \mathrm{ml}$ of viral transport medium (VTM) and placed on ice for processing within 2 hours of collection. Once in the laboratory, swabs were vigorously vortexed to dissociate the cells and mucus and centrifuged for $10 \mathrm{~min}$ at $1600 \mathrm{rpm}$. NP swabs were then rinsed with PBS 2\% FBS, and the cell suspension filtered through $100 \mu \mathrm{m}$ nylon cell strainer (Becton Dickinson) fitted into a $50 \mathrm{ml}$ tube. Cells were washed twice prior to flow cytometry staining. Peripheral blood mononuclear cells (PBMC) were isolated by ficoll density gradient centrifugation using SepMate ${ }^{\mathrm{TM}} 50$ (STEMCELL TM technologies) following the manufacturer's instructions.

Flow cytometry. Cells were stained with viability exclusion dye, together with a panel of pretitrated surface antibodies (Supplementary Table 1) in PBS 2\% FBS on ice for $30 \mathrm{~min}$. The NP swab optimization experiment (Figure 1) included anti-human CD326 (EpCAM) (Biolegend, Clone 9C4) in the surface staining cocktail to stain for epithelial cells. Cells were subsequently washed, permeabilized in fixation/permeabilization solution (eBiosciences) and stained for Ki-67 and perforin. The cells were washed again and resuspended in perm/wash buffer (eBioscience). Data were acquired using a BDLSR Fortessa flow cytometer (BD Biosciences) and analyzed using FlowJo $^{\mathrm{TM}}$ version 10.7.1 (Becton Dickinson Life Sciences).

\section{Dimensionality reduction, visualization and clustering. Flow $\mathrm{Jo}^{\mathrm{TM}}$ version 10.7.1 (Becton}

Dickinson Life Sciences), was utilized to dimensionally reduce and to interrogate immune cell clusters and phenotypes in cells isolated from nasal swabs. Briefly, manual gating was performed to exclude debris, doublets and dead cells. Live CD45+ cells from all participants $(n=21)$ at baseline, visit 2 , and visit 3 , were integrated into a single file which was dimensionally reduced and visualized using t-distributed stochastic neighbour embedding (tSNE), following the default 


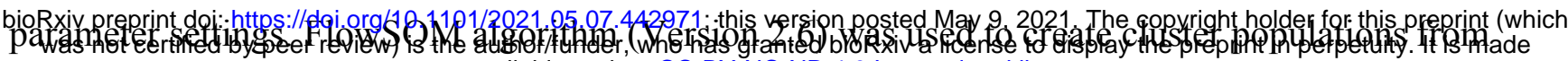
available under ACC-BY-NC-ND 4.0 International license.

the dimensionally reduced data based on their similarity and subsequently segregated based on relative abundance and phenotype using ClusterExplorer (Version 1.5.9).

Antigen-specific stimulation and flow cytometric staining of nasal cells. Approximately 2 months post- $2^{\text {nd }}$ vaccination, participants $(n=15)$ were enrolled. Freshly isolated nasal cells from $(\mathrm{n}=11)$ were stimulated for six hours at $37^{\circ} \mathrm{C} / 5 \% \mathrm{CO}_{2}$ with SARS-CoV-2 spike peptide pools $\mathrm{S}$, $\mathrm{S} 1$ and S+ containing 15-mer sequences overlapping by11 amino acids (PepTivator ${ }^{\circledR}$, Miltenyi Biotec), encompassing the complete spike protein sequence (GenBank MN908947.3) at a final concentration of $1 \mu \mathrm{g} / \mathrm{mL}$ per peptide, in the presence of Golgi-Plug (BD Biosciences), Golgi-Stop (BD Biosciences) and anti-CD107a (clone H4A3, BD Biosciences). As a control for this experiment, $n=4$ participants' cells were left unstimulated. Following stimulation, cells were washed, stained with Live/Dead TM Fixable Aqua viability dye (Invitrogen) for 30 mins on ice. Cells were subsequently washed, permeabilized with fixation/permeabilization solution (eBiosciences) and stained in a cocktail of pre-titrated monoclonal antibodies (Supplementary Table 1). Cells were then washed, acquired using a BDLSR Fortessa (BD Biosciences) and analyzed using FlowJo ${ }^{\mathrm{TM}}$ version 10.7.1 (Becton Dickinson Life Sciences).

Plasma antibody testing. Concentrations of SARS-CoV-2-specific IgG were determined using commercial assays. At visit 3, plasma was diluted 1:10 because of the high magnitude of the responses. IgG recognizing the SARS-CoV-2 spike protein was quantified using the Diasorin Trimeric Spike ELISA, while nucleocapsid antibodies were measured using the ELISA from Abbott.

Statistics. Clinical and demographic data are presented as proportions or medians and interquartile ranges. Linear mixed models were used to compare changes in $\log 10$ transformed immune cell abundance (in nasal mucosa, typically cells/swab; in blood, $\%$ of CD3+CD4+ or CD8+ T cells). In these models, visit as a categorical variable was the main predictor, with visit 2 and 3 compared to baseline. Multivariate models were adjusted for a range of covariates. Raw p-values, beta coefficient and 95\% confidence intervals were reported. Statistical analyses were performed using SPSS v. 27.0 while all the graphing and was performed using Prism version 9 (GraphPad Software, LLC). 